\title{
Implementation to the Different Differential Equations of Homotopy Analysis, Differential Transformed and Adomian Decomposition Method
}

\author{
Necdet Bildik and Şeyma Erdoğan
}

\begin{abstract}
In this study, the concept of Homotopy analysis method (HAM) is briefly introduced. Furthermore some non-linear problems are handled and the solutions of these problems are given using by HAM, DTM, ADM methods and the convergence of the solution is shown to the exact solution. Additionally, the three methods are compared and it is observed that the HAM is more-less efficient and effective than the ADM and DTM in according to the exact solution. In the end, some of the numerical solutions of two examples are presented and the results are shown in graphs and figures.
\end{abstract}

Index Terms-Homotopy analysis method, differential transform method, adomian decomposition method, auxiliary linear operator, embedding parameter, deformation equation.

\section{INTRODUCTION}

The analytical solutions of non-linear problems having strong non-linearity are often difficult to obtain without using the program such as Mathematica, Mapple or software. On semi-analytical methods, the convergence of the solution series most of the time depends on the physical parameters. When the non-linearity is strong, these semi-analytical approaches, often gives unsatisfactory results. The solution of this type of the problems which gives the opportunity to control the region of the convergence and the speed of the solution series.Homotopy analysis method (HAM) is given by Shijun Liao [1]-[5] in 1992. At the same time, this method is the generalization of the non-perturbative method given earlier such as Adomian decomposition method [6], [7]-[11], Lyapunov artificial small parameter method [12], d-expansion method [13], differential transform method [1], [8], [14], [15] and homotopy perturbation method [16]. In other words, this can be thought as a theory of generalized or combined of the previous methods.

\section{Description of the Homotopy Analysis Method}

The homotopy analysis method is generally semi-analytical approach which is used in order to obtain the series solutions of different types of the non-linear equations [3]-[5]. This method used to find the solutions of algebraic equations, ordinary differential equations, integro-differential equations and so on. Unlike the perturbation methods, homotopy analysis method is

Manuscript received April 9, 2013; revised July 30, 2013.

The authors are with Celal Bayar University, Faculty of Arts and Sciences, Department of Mathematics, Muradiye Campus, 45047, Manisa, Turkey (e-mail: necdet.bildik@cbu.edu.tr) independent from small / large physical parameters and also it is not important whether the small / large physical parameters of the problem contains or not for the implementation of the method [17], [18].

Differently from the whole, perturbation methods and the classical non-perturbative methods, the homotopy analysis method provides the possibility to control the region of convergence of the series solution. Homotopy analysis method uses the homotopy that one of the basic concepts of topology similar to homotopy perturbation method [16]. The continuous transformation is formed which carry out the initial approach to the complete solution in order to solve the given equation. The auxiliary linear operator may be chosen to create this type of continuous transformation. Additionally the auxiliary parameter is used in order to ensure the convergence of the series solution. Thus the difficult non-linear problem is converted making use of the homotopyanalysis method to the simple linear sub-problem having infinite number.

For instance, consider a differential equation

$$
A[u(t)]=0
$$

where $A$ is a non-linear operator, $t$ denotes the time, and $u(t)$ is an unkown variable. Let $u_{0}(t)$ is the initial approximation of $u(t)$ and $L$ is an auxiliary linear operator having the property

$$
L f=0 \text { when } f=0 .
$$

We then construct the so-called homotopy $\mathrm{H}[\phi(t ; q) ; q]=(1-q) L\left[\phi(t ; q)-u_{0}(t)\right]+q A[\phi(t ; q)](2.3)$ where $q \in[0,1]$ is an embedding parameter, $\phi(t ; q)$ is the homotopy function. When $q=0$ and $q=1$ then we have

$$
\left.\mathrm{H}[\phi(t ; q) ; q]\right|_{q=0}=L\left[\phi(t ; 0)-u_{0}(t)\right]
$$

And

$$
\left.\mathrm{H}[\phi(t ; q) ; q]\right|_{q=1}=A[\phi(t ; 1)]
$$

Respectively. Using (2.2), it is clear that $\phi(t ; 0)=u_{0}(t)$ is the solution of the equation

$$
\left.\mathrm{H}[\phi(t ; q) ; q]\right|_{q=0}=0
$$

And similarly $\phi(t ; 1)=u(t)$ is also the solution of the equation 


$$
\left.\mathrm{H}[\phi(t ; q) ; q]\right|_{q=1}=0 .
$$

\section{EXAMPLES}

Example 3.1.Let us consider the equation

$$
y^{\prime}=\frac{y \cdot \cos x}{1+2 y^{2}}
$$

Which subject to the initial condition $y(0)=1$.

\section{A. Application of Homogony Analysis Method}

Considering the equation above, then we get

$$
y^{\prime} \cdot\left(1+2 y^{2}\right)-y \cdot \cos x=0 .
$$

which subject to the initial condition $y\left(x_{0}\right)=1$. Additionally let us define the auxiliary linear operator $L[\phi(x ; q)]=\frac{\partial \phi(x ; q)}{\partial x}$ and non-linear operator as

$$
N[\phi(x ; q)]=\frac{\partial \phi(x ; q)}{\partial x}-2 x[\phi(x ; q)]^{2}
$$

And selected the initial condition $y_{0}(x)$. Thus, we write the zero-order deformation equation as

$$
(1-q) \cdot L\left[\phi(x ; q)-y_{0}(t)\right]=q \cdot \hbar \cdot H(x) \cdot N[\phi(x ; q)]
$$

Providing for $q=0$, then $\phi(x ; 0)=y_{0}(x)$

And also

$$
\text { for } q=1 \text {, then } \phi(x ; 1)=y(x)
$$

where $q \in[0,1]$ is an embedding parameter, $\hbar$ is an auxiliary parameter and $H(t) \neq 0$ is an auxiliary function respectively.

On the other hand, let us consider the equations

$$
\begin{aligned}
& y_{m}(x)=\left.\frac{1}{m !} \frac{\partial^{m} \phi(x ; q)}{\partial q^{m}}\right|_{q=0}, \\
& \left.\phi(x ; q)=\phi(x ; 0)+\sum_{m=1}^{+\infty} y_{m}(x) \cdot q^{m}, \vec{y}_{n}(x), y_{1}(x), \ldots, y_{n}(x)\right\}
\end{aligned}
$$

And $m$ th-order deformation equation

$$
L\left[V_{m}(t)-\chi_{m} V_{m-1}(t)\right]=\hbar H(t) R_{m}\left(\vec{V}_{m-1}\right)
$$

which subject to the initial condition

$$
y_{m}(0)=1
$$

Then we may set up the equation

$$
\begin{aligned}
& R_{m}\left[\vec{y}_{m-1}(x)\right]=y_{m-1}{ }^{\prime}(x) . \\
& {\left[1+2 \sum_{i=0}^{m-1} y_{i}(x) \cdot y_{m-1-i}(x)\right]-y_{m-1}(x) \cdot \cos x}
\end{aligned}
$$

Given the different value of $m$ of $1,2,3, \ldots$ respectively, then the iterative solutions are obtained as follows $y_{0}(x)=1$

$y_{1}(x)=1-\hbar \cdot \sin x$

$y_{2}(x)=1+\left(\frac{4 \hbar^{3}+\hbar^{2}}{4}\right)-\left(5 \hbar^{2}+2 \hbar\right) \cdot \sin x-\left(\frac{4 \hbar^{3}+\hbar^{2}}{4}\right) \cdot \cos (2 x)$

\section{B. Application of Differential Transform Method}

Let us consider the same equation (model equation) as

$$
y^{\prime}+2 y^{\prime} y^{2}-y \cos (x)=0
$$

Applying the differential transformation method to the equation (3.1.2), then

$Y(k+1)=\frac{1}{k+1}$

$\left[\sum_{r=0}^{k} Y(r) \cos (k-r)-2\left[\sum_{r=0}^{k}\left(\sum_{r=0}^{k}(r+1) Y(r+1) Y(k-r)\right) Y(k-r)\right]\right]$

is obtained. Thus the iterative solution may be calculated for $k=0,1,2, \ldots$ as follows

$$
Y(1)=\frac{1}{3}, Y(2)=\frac{(1 / 9)+3 \cos 1}{22}, \ldots
$$

Substituting these values in (3.1.3), then the series solution is gained as

$$
y(x)=1+\frac{1}{3} x+\left(\frac{(1 / 9)+3 \cos 1}{22}\right) x^{2}+\ldots
$$

\section{Application of Adomian Decomposition Method}

Let us calculate the approximate solution of the differential equation

$$
y=\cos (x) y-2 y^{2} y^{\prime}
$$

By using Adomian decomposition method which subject to the initial condition $y(0)=1$.

This equation is represented as an operator form as below

$$
L_{x} u=\cos (x) u-2 u^{2} u_{x}=\cos (x) u-2 N(u)(3.1 .4)
$$

where $L_{x}=\frac{\partial}{\partial x}$ and $N(u)=u^{2} u_{x}$ is the non-linear term.

Since the inverse of $L_{x}$ is an integral operator defined by

$$
L_{x}^{-1}=\int_{0}^{x}(.) d x
$$

And applying this inverse operator to both sides of the equation (3.1.4), then

$$
\begin{aligned}
& L_{x}^{-1}\left[L_{x}(x)\right]=L_{x}^{-1}\left[\cos (x) u-2 u^{2} u_{x}\right] \\
& =L_{x}^{-1}[\cos (x) u-2 N(u)]
\end{aligned}
$$




$$
\begin{aligned}
& (3.1 .5) u(x)=u_{0}(x)+L_{x}^{-1}[\cos (x) u-2 N(u)] \\
& A_{0}=F\left(u_{0}\right) \\
& A_{1}=u_{1} F^{\prime}\left(u_{0}\right) \\
& A_{2}=u_{2} F^{\prime}\left(u_{0}\right)+\frac{1}{2} u_{1}^{2} F^{\prime \prime}\left(u_{0}\right) \\
& A_{3}=u_{3} F^{\prime}\left(u_{0}\right)+ \\
& u_{1} u_{2} F^{\prime \prime}\left(u_{0}\right)+ \\
& \frac{1}{3 !} u_{1}^{3} F{ }^{\prime \prime}\left(u_{0}\right) \\
& A_{4}=u_{4} F{ }^{\prime}\left(u_{0}\right)+\left(\frac{1}{2 !} u_{2}^{2}+u_{1} u_{3}\right) \\
& \left.F "\left(u_{0}\right)+\frac{1}{2 !} u_{1}^{2} u_{2} F^{\prime \prime}\left(u_{0}\right)+\frac{1}{4 !} u_{1}^{4} F{ }^{4}\right)\left(u_{0}\right)
\end{aligned}
$$

is obtained.

Since $N(u)=u^{2} u_{x}=\sum_{n=0}^{\infty} A_{n}, \quad$ then the first five terms of the Adomian polynomials $A_{n}$ can be written as

$$
\left\{\begin{array}{l}
u_{0}(x)=1 \\
u_{k+1}(x)=L_{x}^{-1}\left[\cos (x) u_{k}-2 A_{k}\right], k \geq 0
\end{array}\right.
$$

Thus the recurrence relation of the equation (3.1.6) may be written as follows:

Using the recurrence relation (3.1.8) then, the first five terms of the Adomian Decomposition is found as

$$
\begin{aligned}
& u_{0}=1 \\
& u_{1}=\int_{0}^{x}\left[\cos (x) u_{0}-2 A_{0}\right] d x=\sin (x) \\
& u_{2}=\int_{0}^{x}\left[\left(\cos (x) u_{1}-2 A_{1}\right)\right] d x=\frac{\sin (x)^{2}}{2} \\
& u_{3}=\int_{0}^{x}\left[\left(\cos (x) u_{2}-2 A_{2}\right)\right] d x=\frac{\sin (x)^{3}}{6} \\
& u_{4}=\int_{0}^{x}\left[\left(\cos (x) u_{3}-2 A_{3}\right)\right] d x=\frac{\sin (x)^{4}}{24}
\end{aligned}
$$

Therefore the solution using ADM is obtained as $u(x, t)=\sum_{n=0}^{\infty} u_{n}(x, t)=u_{0}+u_{1}+u_{2}+u_{3}+u_{4}+\cdots$ $=1+\sin (x)+\frac{\sin (x)^{2}}{2}+\frac{\sin (x)^{3}}{6}+\frac{\sin (x)^{4}}{24}+\cdots$

$\mathrm{O}$ the other hand, the analytical solution of the given equation is calculated as

$$
\ln |y|+y^{2}=\sin x+1
$$

It is very interesting that the analytic solution of this equation is exactly the same of approximate solution by coencident. Now let us compare, the approximate solution of non-linear differential equation by using homotopy analysis method (HAM), differential transform method (DTM) and Adomian decomposition method (ADM) with exact solution for $x=0.1$ and depicts the figures in two and three dimensional graphics.

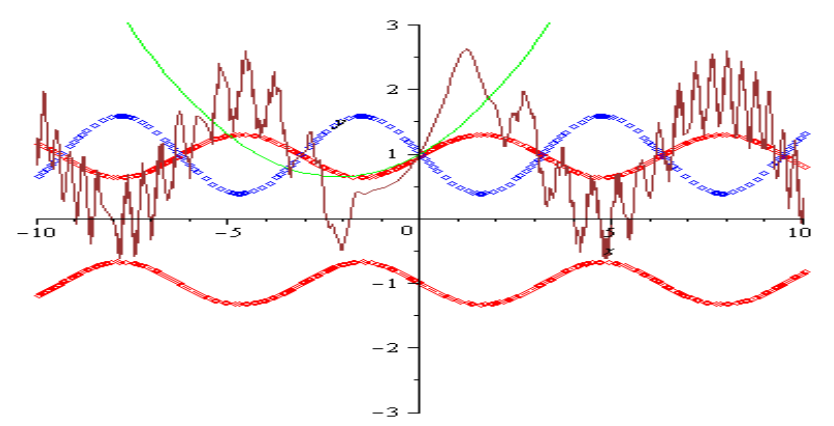

Fig. 3.1. The graph of the exact solution is shown by red color, the homotopy analysis solution is shown by blue color, the DTM solution is shown by green color and the Adomian solution is shown by brown color.

Three-dimensional graphics of the solutions are shown as follows
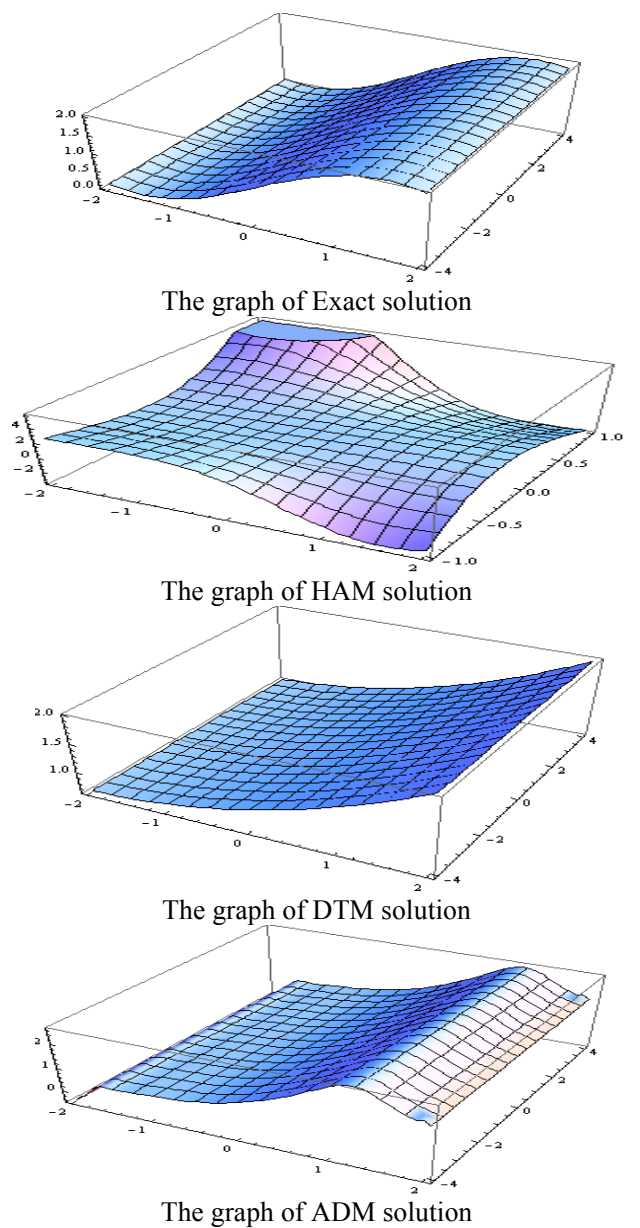

Example 3.2.Let us consider the equation

$$
y^{\prime}=2 x y^{2}
$$

which subject to the initial condition $y(0)=1$.

\section{Application of Homotopy Analysis Method}

If we select the initial approach $y_{0}(x)=x^{2}+1$ and 
additionally define the auxiliary linear operator

$$
\begin{aligned}
L[\phi(x ; q)] & =\frac{\partial \phi(x ; q)}{\partial x} \text { and non-linear operator as } \\
{[\phi(x ; q)] } & =\frac{\partial \phi(x ; q)}{\partial x} \cdot\left[1+2[\phi(x ; q)]^{2}\right]-\phi(x ; q) \cdot \cos x
\end{aligned}
$$

then, we write the following, zero-order deformation equation

$$
(1-q) \cdot L\left[\phi(x ; q)-y_{0}(t)\right]=q \cdot \hbar \cdot H(x) \cdot N[\phi(x ; q)]
$$

providing

and also

$$
\text { for } q=0 \text {, then } \phi(x ; 0)=y_{0}(x)
$$

$$
\text { for } q=1, \text { then } \phi(x ; 1)=y(x)
$$

where $q \in[0,1]$ is an embedding parameter, $\hbar$ is an auxiliary parameter and $H(t) \neq 0$ is an auxiliary function respectively.

On the other hand, let us consider the equations

$$
\begin{aligned}
& y_{m}(x)=\left.\frac{1}{m !} \frac{\partial^{m} \phi(x ; q)}{\partial q^{m}}\right|_{q=0}, \\
& \phi(x ; q)=\phi(x ; 0)+ \\
& \sum_{m=1}^{+\infty} y_{m}(x) \cdot q^{m}, \\
& \vec{y}_{n}(x)=\left\{y_{0}(x), y_{1}(x), \ldots, y_{n}(x)\right\}
\end{aligned}
$$

and $m$ th-order

deformation equation

$$
L\left[V_{m}(t)-\chi_{m} V_{m-1}(t)\right]=\hbar H(t) R_{m}\left(\vec{V}_{m-1}\right)
$$

then, we may set up the equation

$$
\begin{aligned}
& R_{m}\left[\vec{y}_{m-1}(x)\right]=y_{m-1}^{\prime}(x)- \\
& 2 x \cdot \sum_{i=0}^{m-1} y_{i}(x) \cdot y_{m-1-i}(x)
\end{aligned}
$$

which subject to the initial condition $y_{m}(0)=1$. Given the different value of $m$, then the iterative solutions are obtained as follows

$$
\begin{aligned}
& y_{0}(x)=x^{2}+1 \\
& y_{1}(x)=1-\hbar x^{4}-\frac{\hbar}{3} x^{6} \\
& y_{2}(x)=1-2 \hbar x^{2}-\left(\hbar^{2}+\hbar+1\right) x^{4} \\
& +\left(\frac{\hbar^{2}-\hbar}{3}\right) x^{6}+\frac{2 \hbar^{2}}{3} x^{8}+\frac{2 \hbar^{2}}{15} x^{10}
\end{aligned}
$$

Application of Differential Transform Method Let usconsider the same equation (model equation) as

$$
y^{\prime}-2 x y^{2}=0
$$

$$
\begin{aligned}
& Y(k+1)=\frac{1}{k+1} \\
& {\left[\sum_{r=0}^{k} Y(r) \cos (k-r)-2\left[\sum_{r=0}^{k}\left(\sum_{r=0}^{k}(r+1) Y(r+1) Y(k-r)\right) Y(k-r)\right]\right]}
\end{aligned}
$$

Applying the differential transformation method to the equation (3.2.2), then

Is obtained. Thus the iterative solution may be calculated for $k=0,1,2, \ldots$ as follows

$$
Y(1)=0, Y(2)=4, Y(3)=0, Y(4)=32, \ldots
$$

Substituting these values in (3.2.3), then the series solution is found as

$$
y(x)=1+4 x^{2}+32 x^{4}+\ldots
$$

\section{E. Application of Adomian Decomposition Method}

Let us calculate the approximate solution of the differential equation $y^{\prime}=2 x y^{2}$ by using Adomian decomposition method subject to the initial condition $y(0)=1$. This equation is represented as an operator form such as

$$
L_{x} u=2 x u^{2}=2 x N(u)
$$

where $L_{x}=\frac{\partial}{\partial x}$ and $N(u)=u^{2}$ is the non-linear term.

Since the inverse of $L_{x}$ is an integral operator defined by

$$
L_{x}^{-1}=\int_{0}^{x}(.) d x
$$

And applying this inverse operator to both sides of the equation (3.2.4), then

$$
\begin{array}{r}
L_{x}^{-1}\left[L_{x}(x)\right]=L_{x}^{-1}\left[2 x u^{2}\right]=L_{x}^{-1}[2 x N(u)](3.2 .5) \\
u(x)=u_{0}(x)+L_{x}^{-1}\left[2 x u^{2}\right]=u_{0}(x)+L_{x}^{-1}[2 x N(u)]
\end{array}
$$

is obtained.

Since $N(u)=u^{2}=\sum_{n=0}^{\infty} A_{n}, \quad$ then the first five terms of the Adomian polynomials $A_{n}$ can be written as

$$
\begin{aligned}
& A_{0}=F\left(u_{0}\right) \\
& A_{1}=u_{1} F^{\prime}\left(u_{0}\right) \\
& A_{2}=u_{2} F^{\prime}\left(u_{0}\right)+\frac{1}{2} u_{1}^{2} F^{\prime \prime}\left(u_{0}\right) \\
& A_{3}=u_{3} F^{\prime}\left(u_{0}\right)+u_{1} u_{2} F^{\prime \prime}\left(u_{0}\right) \\
& +\frac{1}{3 !} u_{1}^{3} F^{\prime \prime}\left(u_{0}\right) \\
& A_{4}=u_{4} F^{\prime}\left(u_{0}\right)+\left(\frac{1}{2 !} u_{2}^{2}+u_{1} u_{3}\right) F^{\prime \prime} \\
& \left(u_{0}\right)+\frac{1}{2 !} u_{1}^{2} u_{2} F^{\prime \prime}\left(u_{0}\right)+\frac{1}{4 !} u_{1}^{4} F(4)\left(u_{0}\right)
\end{aligned}
$$

Thus the recurrence relation of the equation (3.2.6) may be written as follows

$$
\left\{\begin{array}{l}
u_{0}(x)=1 \\
u_{k+1}(x)=L_{x}^{-1}\left[\left(2 x A_{k}\right)\right], k \geq 0
\end{array}\right.
$$


Using the recurrence relation (3.2.8) then, the first five terms of the Adomian decomposition are found as

$$
\begin{aligned}
u_{0} & =1 \\
u_{1} & =\int_{0}^{x}\left[\left(2 x A_{0}\right)\right] d x=\int_{0}^{x}\left[\left(2 x u_{0}^{2}\right)\right] d x=x^{2} \\
u_{2} & =\int_{0}^{x}\left[\left(2 x A_{1}\right)\right] d x=\int_{0}^{x}\left[\left(2 x\left(2 u_{0} u_{1}\right)\right)\right] d x=x^{4} \\
u_{3}=\int_{0}^{x}\left[\left(2 x A_{2}\right)\right] d x=\int_{0}^{x}\left[\left(2 x\left(2 u_{0} u_{2}+u_{1}^{2}\right)\right)\right] d x=x^{6} & \int_{0}^{x}\left[\left(2 x A_{3}\right)\right] d x=\int_{0}^{x}\left[\left(2 x\left(u_{2}^{2}+2 u_{1} u_{2}\right)\right)\right] d x=x^{8}
\end{aligned}
$$

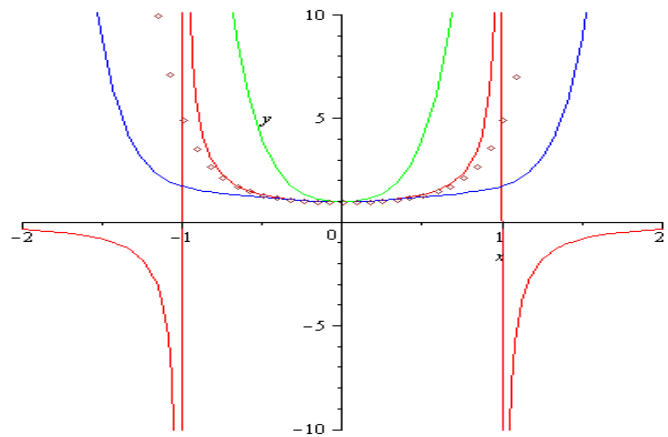

Fig. 3.2. The graph of the exact solution is shown by red color, the Homotopy analysis solution is shown by blue color, the DTM solution is shown by green color and the Adomian solution is shown by pink color

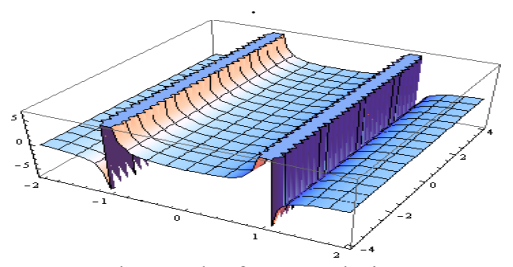

The graph of exact solution

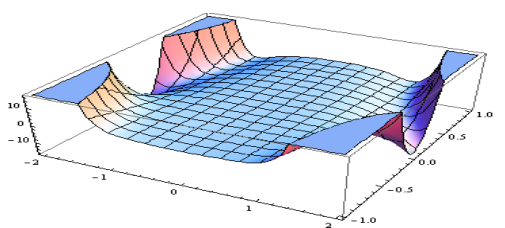

The graph of HAM solution

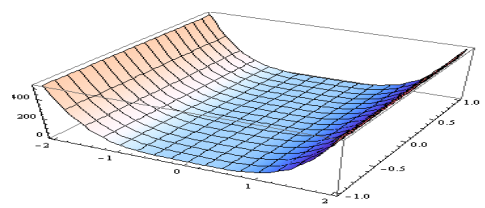

The graph of DTM solution

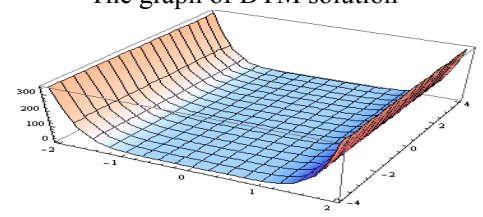

The graph of ADM solution
On the other hand, the analytical solution of the given equation is calculated as

$$
y=\left(1-x^{2}\right)^{-1}
$$

Here, again the analytic solution of given equation is exactly the same of approximate solution by coencident. Now let us compare, the approximate solution of non-linear differential equation by using Homotopy analysis method (HAM), Differential transform method (DTM) and Adomian decomposition method (ADM) with exact solution for $x=0.1$ and are depicts the figures in two and three dimensional graphics.

Three-dimensional graphics of solutions are shown as follows

\section{CONCLUSION}

Unlike all previous analytic techniques, the Homotopy analysis method provides us with great freedom to express solutions of a given non-linear problem by means of different base functions. Therefore we can approximate a non-linear problem more efficiently by choosing a proper set of base functions. This is because the convergence region and rate of series are chiefly determined by the base functions used to express the solution.

Therefore, most of the time this method gives the best approximation compare to the exact solution as well as the other approximation methods concern. We need again only focus on choosing proper initial approximations, auxiliary linear operators, auxiliary functions and proper values of $\hbar$ to ensure that solution series convergence.

\section{REFERENCES}

[1] S. J. Liao, "Comparison between the homotopy analysis method and homotopy perturbation method," Appl. Math. Comput, vol. 169, 2005.

[2] S. J. Liao, "An explicit, totally analytic approximation of Blasius' viscous flow problems," Int. J. Non-Linear Mech, vol. 34, pp. 759-778, 1999.

[3] S. J. Liao, "The proposed homotopy analysis technique for the solution of non-linear problems," Ph. D. Thesis, Shanghai Jiao Tong University, 1992.

[4] S. J. Liao, Beyond Perturbation: Introduction to the homotopy analysis method, Chapman and Hall/CRC Press, Boca Raton, 2003.

[5] S. J. Liao, "On the homotopy analysis method for non-linear problems," Appl. Math. Comput, vol. 47, pp. 499-513, 2004.

[6] H. Bayramoğlu, "The solution with decomposition method of wave equations," Master's Thesis, Celal Bayar University, Manisa/TURKEY, 2001.

[7] N. Bildik, A. Konuralp, F. O. Bek, and S. Küçükarslan, "Solution of different type of the partial differential equation by differential transform method and Adomians decomposition method," Appl. Math. Comput, vol. 172, pp. 551-567, 2006.

[8] N. Bildik and A. Konuralp, "Two-dimensional differential transform method, Adomian's decomposition method, and variational iteration method for partial differential equations," International Journal of Computer Mathematics, vol. 83, vol. 12, pp. 973-987, 2006.

[9] G. Adomian, "Convergent series solution of non-linear equations," Journal of Computational and Applied Mathematics, vol. 11, pp. 225-230, 1984.

[10] G. Adomian, "On the convergence region for decomposition solutions," Journal of Computational and Applied Mathematics, vol. 11, pp. 379-380, 1984.

[11] G. Adomian, "A review of the decomposition method in applied mathematics," Journal of Computational and Applied Mathematics, vol. 135, pp. 501-544, 1988.

[12] A. M. Lyapunov, "General problem on stability of motion (English translation)," Taylor and Francis, London, 1992. 
[13] A. V. Karmishin, A. I. Zhukov, and V. G. Kolosov, "Methods of dynamics calculation and testing for thin-walled structures," Mashinostroyenie, Moscow, 1990.

[14] M. J. Jang, C. L. Chen, and Y. C. Liu, "Two-dimensional differential transform for partial differential equations," Appl. Math. Comput, vol. 121, pp. 261-270, 2001.

[15] M. J. Jang and C. L. Chen, "Analysis of the response of strongly non-linear damped system using differential transformation technique," Appl. Math. Comput, vol. 88, pp. 137-151, 1997.

[16] A. M. Siddiquia, R. Mahmoodb, and Q. K. Ghorib, "Homotopy perturbation method for thin film flow of a fourth grade fluid down a vertical cylinder," Phys. Lett. A, vol. 352, pp. 404-410, 2006.

[17] M. Ayub, A. Rasheed, and T. Hayat, "Exact flow of a third grade fluid past a porous plate using homotopy analysis method," Int. J. Eng. Sci., vol. 41, pp. 2091-2103, 2003.

[18] A. H. Nayfeh, Perturbation methods, John Wiley and Sons, New York, 2000.

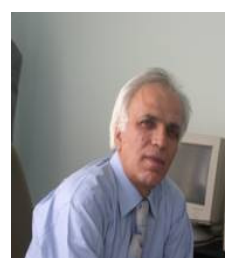

Necdet Bildik was born in Sivas, Turkey in 1951. He graduated from faculty of science, department of mathematics, and Ankara University in 1974 . He completed his MSc.degree in University of Louisville, Kentucky, USA in 1978. He completed his doctorate in Oklahoma State University in 1982. He was assistant professor in 1988 and was associate professor in 1995. He was become professor in 2003. He is interested in numeric analysis, ordinary and partial differential equations, real and complex dynamical systems, and ergodic and stability theory.

He has thirty four published articles in the national and international journals. His forty eight papers have been presented in national and international conferences. He also completed one of main scientific research projects at Celal Bayar University in 2006.

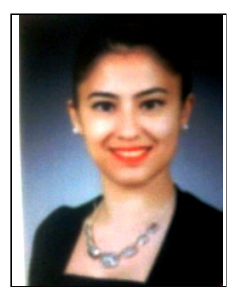

Şeyma Erdoğan was born in İskenderun, Turkey in 1987. She was graduated with BSc. degree from Celal Bayar University, faculty of art and sciences, department of mathematics in 2009. She was worked on homotopy analysis method for the homogeneous and non-homogeneous differential equations and also compared with someof the well-known methods of among them. She completed her MSc.thesis atCelal Bayar University, Turkey in 2012. 